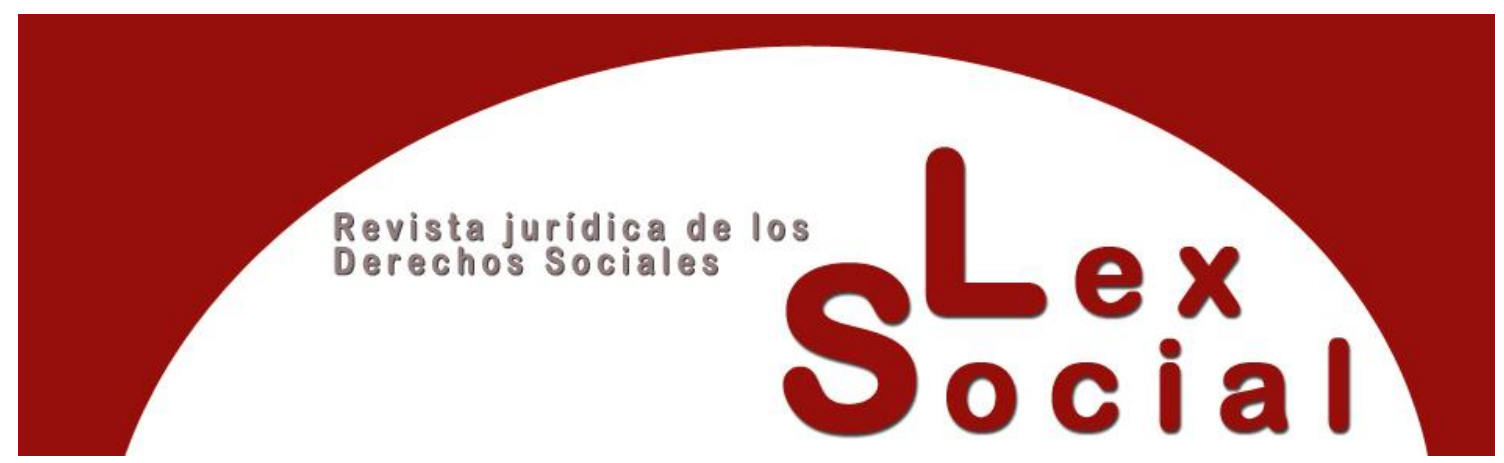

\title{
EL TRATAMIENTO DEL DESEMPLEO EN EL DERECHO DERIVADO DE LA UNIÓN EUROPEA
}

\section{THE TREATMENT OF UNEMPLOYMENT IN SECONDARY LAW OF THE EUROPEAN UNION}

\author{
JOSÉ MANUEL PAZÓ ARGIBAY \\ Universidad de Santiago de Compostela \\ https://orcid.org/0000-0003-3718-0247
}

Cómo citar este trabajo: Pazó Argibay, J.M. (2021). El tratamiento del desempleo en el derecho derivado de la Unión Europea. Lex Social, Revista De Derechos Sociales, 11 (2), 400-421. https://doi.org/10.46661/lexsocial.5923

\section{RESUMEN}

El tradicional carácter económico de los Tratados relegó a un segundo plano el desarrollo de los aspectos sociales, entendidos estos como resultado natural de la cuestión económica. En consecuencia, la principal vía de desarrollo en materia social en el ámbito del Derecho de la Unión Europea ha sido a través de instrumentos de Derecho derivado.

En relación con el desempleo, su desarrollo normativo ha seguido el mismo esquema, con una presencia residual en el Derecho originario de la Unión Europea y su necesario y tardío desarrollo a través de instrumentos de derecho derivado.

Este trabajo ofrece un recorrido sobre los principales hitos en el desarrollo normativo del desempleo en el Derecho derivado de la Unión Europea.

Palabras Clave: Desempleo, Unión Europea, Política Social, Seguridad Social. 


\begin{abstract}
Traditional economic character of Treaties relegated the social aspects, understood as a natural result of the economic question. As a result, the development of social question in the European Union law has been through secondary law.

Unemployment regulation development has followed the same pattern, with a residual presence in the primary EU law and its necessary development through secondary law.

This paper offers a tour of the main landmarks in the unemployment normative development in secondary EU law.
\end{abstract}

KEYWORDS: Unemployment, European Union, Social Policy, Social Security

SUMARIO

I. La residual presencia del desempleo en el Derecho originario de la Unión Europea

II. El desempleo en el Derecho derivado de la Unión Europea

III. Conclusiones

\title{
I. La residual presencia del desempleo en el Derecho originario de la Unión europea
}

Si hay un adjetivo que puede resumir a la perfección la presencia de la cuestión social en general, y del desempleo en particular, en el Derecho originario de la Unión Europea, este sería, si lugar a dudas, el de residual.

La política social no ha sido cuestión prioritaria en los Tratados y, por lo general, su tratamiento ha estado siempre relegado a un segundo plano por las cuestiones económicas $^{1}$ y políticas, unas veces como medio, otras como consecuencia ${ }^{2}$. La presencia de la cuestión del desempleo en los Tratados ha sido, por lo general, de mero contenido declarativo ${ }^{3}$. Habida cuenta de la finalidad claramente económica de las

\footnotetext{
1"The only role to be acknowledged for labour law is ancillary". SCIARRA, Silvana, "Social Values and the Multiple Sources of European Social Law”, European Law Journal, 1995, Vol. 1, n¹, p. 65.

${ }^{2}$ No obstante, BLÁZQUEZ AGUDO llama la atención sobre la necesidad de no desatender lo social para lograr lo económico. Sobre su necesaria interrelación, véase BLÁZQUEZ AGUDO, Eva María, "El futuro de la libre circulación de trabajadores. Repensando su contenido a partir de la Directiva Marco sobre mercado interior", Revista del Ministerio de Trabajo y Asuntos Sociales, 2006, n 62, pp. 111-113.

${ }^{3}$ Como señala GARCÍA MURCIA "no se advierte aún como terreno practicable para una intervención directa por parte de la Comunidad, entre otras razones por su intenso enraizamiento en las políticas nacionales". GARCÍA MURCIA, Joaquin, "Desempleo", Ponencia General del XIV Congreso Nacional
} 
primeras etapas de lo que hoy conocemos como Unión Europea, lo social tuvo siempre una presencia testimonial y, en todo caso, supeditada a la prioridad económica. Todo ello, sobre el errado convencimiento de que el desarrollo social devendría de manera natural como consecuencia del desarrollo económico. En consecuencia, lo prioritario fue actuar sobre lo económico.

Los avances producidos en esta materia no han tenido una fácil entrada en el cuerpo normativo de los Tratados, teniendo que optarse en un primer momento por vías alternativas. Por ejemplo, debe recordarse que el mayor paquete de contenido social del derecho originario tuvo que acomodarse en un primer momento en un Protocolo anexo al Tratado de Maastricht, siendo plenamente incorporado años después por el Tratado de Amsterdam. Del mismo modo, ha sido también frecuente el uso de fórmulas de exclusión voluntaria ante disposiciones de carácter social, especialmente por parte del Reino Unido 4 .

Pese a todo, no debe desmerecerse la innovadora propuesta de Aristide Briand en el año 1929, hablando ya de una Unión Europea o Asociación Europea ${ }^{5}$; la Conferencia de la Haya ${ }^{6}$ cuya resolución económica y social abogaba ya por promover medidas que favoreciesen la movilidad de la mano de obra en la mayor medida posible o la coordinación de la legislación social para poder dar cumplimiento a lo anterior ${ }^{7}$; la Carta Social Europea, que abogaba por el establecimiento de servicios gratuitos de empleo para todos los trabajadores, así como la promoción de la orientación, la formación y la readaptación profesional ${ }^{8}$; el artículo 69 de la CECA, que eliminaba cualquier tipo de restricción por motivos de nacionalidad con respecto a cualquier cuestión de empleo y que, en su apartado 4, disponía a los Estados la necesidad de adopción de las medidas necesarias a fin de que las disposiciones de Seguridad Social no constituyeran un obstáculo para los movimientos de mano de obra; el TCEE, con sus artículos 48 a 51, relativos a la libre circulación de trabajadores y a la coordinación de las legislaciones nacionales garantizando la acumulación de todos los períodos tomados en consideración por las distintas legislaciones nacionales para adquirir y/o conservar el derecho a las prestaciones sociales, así como para su cálculo; o los

de Derecho del Trabajo y de la Seguridad Social, Madrid, Ministerio de Trabajo y Asuntos Sociales, Colección Informes y Estudios, Serie Relaciones Laborales, 2004, nº 61, p. 57.

${ }^{4}$ NIELSEN, R, EU Labour Law, Copenhagen, DJOF Publishing, 2013, p. 48.

${ }^{5}$ MANGAS MARTÍN, Araceli, LIÑÁN NOGUERAS, Diego Javier, Instituciones y Derecho de la Unión Europea, Madrid, Editorial Mc Graw Hill, 1996, p. 5.

${ }^{6} 1948$.

7 Resolución Económica y Social del Congreso de La Haya (7-10 de Mayo de 1948). http://www.cvce.eu/en/obj/economic_and_social_resolution_of_the_hague_congress_7_10_may_1948en-537c7689-d702-4a15-88bd-4db51fd07b3a.html

${ }^{8}$ Sobre su "laboriosa gestación" y contenido social, RODRÍGUEZ-PIÑERO y BRAVO-FERRER, Miguel, "La Carta Social Europea y su puesta en práctica", Revista de Instituciones Europeas № 5, 1978, pp. $53-69$. 
artículos 123 y siguientes, por el que se creaba el FSE como instrumento para fomentar las oportunidades de empleo y movilidad geográfica y profesional de los trabajadores ${ }^{9}$.

Tan sólo preocupante nivel de desempleo en Europa a finales de los años ochenta y principios de los noventa hizo reaccionar a los Estados ante la evidencia de que las acciones centradas únicamente sobre los aspectos económicos no aportaban los resultados esperados sobre el desarrollo social y sobre el empleo en particular, por lo que la cuestión social comenzó a ganar presencia, si bien su desarrollo fue ciertamente controvertido. La limitación competencial de la Unión en esta materia, unido a las recelosas políticas estatales, provocaron un desarrollo lento y desigual de la cuestión social. Así, mientras que las cuestiones relativas a la libre circulación de trabajadores experimentaron un importante y cohesionado desarrollo, los aspectos de Seguridad Social y protección social han estado siempre estrechamente atados a la regla de la unanimidad.

En este sentido, cabe destacar el Protocolo XIV y su correspondiente Acuerdo sobre Política Social, anexo al Tratado de Maastricht ${ }^{10}$ que, si bien introducía expresamente el mecanismo de la mayoría cualificada del Consejo para la adopción de determinadas disposiciones de carácter social ${ }^{11}$, mantenía la exigencia de la unanimidad en cuestiones relativas a la Seguridad Social y la protección social de los trabajadores, lo que provocaba cierto efecto frenada ${ }^{12}$, el Tratado de Amsterdam ${ }^{13}$, que supuso la plasmación, de manera formal, de la preocupación por el desempleo en el cuerpo del $\mathrm{TCE}^{14}$ y que introdujo seis nuevos artículos a través de los cuales la cuestión del empleo entró plena y formalmente en el Tratado ${ }^{15}$; la Carta de Derechos Fundamentales de la Unión Europea ${ }^{16}$, que en su artículo 34 reconocía el derecho al acceso de prestación de Seguridad Social y garantizaba esta protección en casos como, entre otros, situaciones de pérdida de empleo; el Tratado de Niza ${ }^{17}$, que ampliaba ámbitos de actuación en materia social ${ }^{18}$ pero mantenía la regla de la unanimidad para el ámbito de la Seguridad Social y la protección social de los

${ }^{9}$ En mayor profundidad sobre su naturaleza, regulación, objetivos y funcionamiento véase GÁRATE CASTRO, Javier, “Apuntes sobre la regulación y funcionamiento del Fondo Social Europeo", Revista Española de Derecho del Trabajo nº 30, 1987, pp. 243-267.

10 Año 1992.

11 Ampliando así el abanico de materias de índole social susceptibles de ser adoptadas por esta vía.

12 RODRÍGUEZ-PIÑERO ROYO, Miguel, CASTELLANO BURGUILLO, Emilia, "La política de empleo de la Unión Europea”, Trabajo: Revista Andaluza de Relaciones Laborales n 10, 2001, p. 18.

13 Año 1997.

${ }^{14}$ Con la incorporación del Acuerdo de Política Social del Tratado de Maastricht dentro de su articulado, así como con la creación de un Título específico sobre Empleo, el Título VI bis.

15 Por ejemplo, el artículo 109S creaba el Comité de Empleo y los artículos 117 a 120 contenían el Acuerdo de Política Social de Maastricht.

${ }^{16}$ Año 2000.

17 Año 2001.

18 La lucha contra la exclusión y modernización de sistemas de protección social. 
trabajadores; o el Tratado de Lisboa ${ }^{19}$, cuyo artículo 5 precisó un poco más y diferenció entre políticas de empleo y políticas sociales, dando mayor intensidad a las primeras $^{20}$ frente a las segundas ${ }^{21}$ e incorporando en su artículo 6 las disposiciones de la Carta de los Derechos Fundamentales de la Unión Europea ${ }^{22}$.

En definitiva, la presencia del desempleo en los Tratados ha sido, por lo general, de mero contenido declarativo. Solo a partir de finales de los años 80 y principios de los 90 la cuestión social comenzó a ganar presencia, si bien su desarrollo fue ciertamente asimétrico y controvertido ${ }^{23}$.

Como se expondrá a continuación, la principal vía de desarrollo en materia de desempleo en el ámbito del Derecho de la Unión Europea ha sido a través de instrumentos de Derecho derivado. Esto ayudó, por un lado, a clarificar y garantizar determinados derechos y, por otro, a adaptarse a las nuevas realidades del mercado laboral europeo. En relación con el desempleo, mención aparte de los Reglamentos de coordinación, las acciones en esta materia tomaron forma, mayormente, de políticas activas, centrando fundamentalmente su intervención en el fomento del empleo y la armonización de las legislaciones estatales.

\section{EI desempleo en el Derecho derivado de la Unión Europea}

Como se ha anticipado, el artículo 51 TCEE, en su redacción original de 1957, encomendaba al Consejo, a propuesta de la Comisión, la adopción de cuantas medidas fuesen necesarias para garantizar la libre circulación de trabajadores. En concreto, el artículo 51 TCEE mencionaba la necesidad de arbitrar instrumentos que garantizasen, por un lado, la acumulación de todos los períodos tomados en consideración por las distintas legislaciones nacionales para adquirir, conservar y calcular prestaciones sociales y, por otro, el pago de prestaciones a las personas que residan en los territorios de los Estados miembros.

En aplicación de este artículo, vieron la luz el Reglamento $n^{\circ} 3^{24}$, de 25 de septiembre, que contenía las disposiciones en materia de Seguridad Social de los trabajadores

\footnotetext{
${ }^{19}$ Año 2007.

20 “ la Unión tomará medidas".

21 "la Unión podrá tomar iniciativas para garantizar la coordinación de las políticas de empleo de los Estados miembros".

${ }^{22}$ Aunque manteniendo la exigencia de la unanimidad para los ámbitos de Seguridad Social y protección social de los trabajadores.

23 Recurriendo a anexos, fórmulas de exclusión voluntaria, etc.

${ }^{24}$ Reglamento $\mathrm{n}^{\mathrm{o}} 3$, de 25 de septiembre, sobre la seguridad social de los trabajadores migrantes. DO $\mathrm{n}^{\mathrm{o}}$ 30 de 16/12/1958, p. 561/58.
} 
migrantes, y el Reglamento $n^{\circ} 4^{25}$, de 3 de diciembre, con disposiciones de aplicación del anterior, conocidos como los Reglamentos de Coordinación. Ambos eran herederos directos del Convenio Europeo sobre la Seguridad Social de los Trabajadores Migrantes, firmado el 9 de diciembre de 1957 al amparo del artículo 69 TCECA $^{26}$. Apenas unos meses antes de la firma de este Convenio de Seguridad Social en el marco de la CECA, se firmó el TCEE, en cuyo artículo 51 se encomendaba al Consejo una tarea similar a la que el artículo 69.4 TCECA encomendaba a los Estados miembros, esto es, la adopción de medidas en el ámbito de la Seguridad Social que garantizasen la libre circulación de los trabajadores. Entendiendo que lo establecido en el Convenio de Seguridad Social se adaptaba al mandato del artículo 51 TCEE, se optó por convertir el Convenio de Seguridad Social de la CECA en el Reglamento $n^{\circ} 3^{27}$. Al mismo tiempo, el documento administrativo que se había elaborado para la aplicación práctica del Convenio de Seguridad Social se adaptó en lo que pasó a ser el Reglamento no 4.

El Reglamento $\mathrm{n}^{\mathrm{o}} 3$ se aplicaba, siguiendo lo establecido en su artículo 2, a la legislación en materia de prestaciones por desempleo ${ }^{28}$. En concreto, las cuestiones relativas al desempleo se abordaron en los seis artículos (33 a 38) que componían el Capítulo 6. El artículo 33 regulaba la totalización de períodos, estableciendo en su apartado 1 que, para la adquisición, mantenimiento o recuperación de determinados derechos, el trabajador que hubiera estado sometido a la legislación de varios Estados miembros, de manera sucesiva o alternativa, tendría derecho a la totalización de tales períodos de cotización siempre y cuando estos no estuvieran superpuestos. En el caso de que un Estado miembro condicionase la percepción de prestaciones de carácter contributivo al hecho de acreditar periodos de cotización previa, o períodos asimilados, el Estado competente admitiría como tales, en la medida en que fuese necesario, los períodos de cotización, o asimilados, generados en otros Estados miembro, siempre y cuando estos períodos fuesen iguales a los exigidos para los trabajadores del primer Estado.

De la misma forma, para el caso de aquellas prestaciones de carácter no contributivo en las que se exigiese un período de empleo o residencia previa, un Estado miembro

\footnotetext{
25 Reglamento $\mathrm{n}^{\mathrm{o}}$ 4, de 3 de diciembre, por el que se establecen disposiciones de aplicación y se completan las disposiciones del Reglamento $\mathrm{n}^{\mathbf{o}} 3$ sobre la seguridad social de los trabajadores migrantes. DO n 30 de 16/12/1958, p. 597/58.

${ }^{26}$ Artículo 69.4 TCECA: "Los Estados miembros [...] tratarán de buscar entre sí cuantas soluciones sigan siendo necesarias a fin de que las disposiciones relativas a la seguridad social no constituyan un obstáculo para los movimientos de la mano de obra". Todo ello, con la debida mención y poniendo en valor al primigenio acuerdo de Seguridad Social de los bateleros del Rhin. En detalle LAMBEAUX, O, « L'accord international de sécurité sociale applicable aux bateliers du Rhin », Revista Droit Social, 1957, $\mathrm{n}^{\circ}$ 9-10, pp. 510-518.

27 Sobre los trabajos de elaboración del Convenio de Seguridad Social en el marco de la CECA y su posterior conversión en el Reglamento n 3 véase RIBAS, Jacques Jean, JONCZY, Marie Jose, SÉCHÉ, Jean Claude, Derecho Social Europeo, Madrid, Instituto de Estudios Sociales, Ministerio de Trabajo, 1980, pp. 219-221.

${ }^{28}$ Artículo 2, apartado 1, letra g).
} 
tendría en cuenta, en la medida en que fuese necesario, los períodos de empleo y residencia en otros Estados miembros como si estos fuesen obtenidos bajo la legislación del primer Estado. En relación con los períodos de residencia a efectos de determinados beneficios o prestaciones sociales, aquellos Estados que condicionasen estos beneficios a un período concreto de residencia, no podrían requerir un período de residencia a los trabajadores provenientes de otro Estado miembro superior al requerido para los ciudadanos del propio Estado.

Por su parte, el artículo 34 del Reglamento $n^{\circ} 3$ pretendía garantizar la igualdad en el cálculo de aquellas prestaciones que estaban condicionadas al salario previo, estableciendo que, si en base a la legislación de un Estado miembro, la cuantía de la prestación variaba con respecto al salario previo, la institución competente tendría en cuenta para el cálculo, en sustitución del la cantidad de salario que el trabajador percibía en el otro Estado, el salario medio para una ocupación análoga o equivalente en el lugar de residencia del desempleado. Asimismo, el apartado segundo del artículo 34 establecía que, si en base a la legislación de un Estado miembro, la cuantía de la prestación estaba ligada al número de familiares, aunque no conviviesen con el beneficiario, la institución competente debería tener en cuenta para el cálculo de la prestación a todos los familiares del interesado, aunque estos viviesen en el territorio de otro Estado miembro distinto del Estado competente.

El artículo 35 del Reglamento $n^{\circ} 3$ regulaba la situación de aquellos desempleados que, una vez reconocida una prestación en virtud de la legislación de un Estado miembro, cambiaban su residencia al territorio de otro Estado. En este caso, el Reglamento garantizaba la conservación de los derechos adquiridos, si bien condicionados a unos plazos máximos. Además, el mantenimiento de la prestación debía ser autorizada por acuerdo entre la institución competente y la institución del nuevo Estado de residencia del beneficiario, estableciendo a su vez una serie de situaciones por las cuales esta autorización no podría ser rechazada. Por su parte, los artículos 36, 37 y 38 establecían reglas de aplicación limitada a determinados colectivos de trabajadores, así como un sistema de reembolso de prestaciones por desempleo entre Estados.

A su vez, el Reglamento $\mathrm{n}^{\mathrm{o}} 4$ fue concebido como un instrumento para la aplicación y como complemento del anterior. En relación con el desempleo, su contenido de desarrollaba en el capítulo 5, en los artículos 62 a 66. A este respecto, por ejemplo, el artículo 62 extendía las reglas generales para la totalización de períodos a efectos de la prestación por desempleo. El artículo 63 recogía la necesidad de que el interesado en hacer valer alguno de los supuestos del artículo 33 de Reglamento $n^{\circ} 3$ debía presentar ante la institución competente un certificado relativo a los períodos a tener en cuenta para, en caso de que fuese necesario, completar los períodos acreditados bajo la legislación aplicada por esa institución, si bien esta certificación podía remitirse directamente entre instituciones en los supuestos en los que no se presentaba por parte de la persona interesada. Igual petición se contemplaba en el artículo 64 para todas 
aquellas personas que pretendían hacer valer el derecho recogido en el artículo 34 del Reglamento $\mathrm{n}^{\mathrm{o}} 3$ al serle requerida certificación de la institución de origen relativa a la profesión u ocupación ejercida en el Estado de origen antes del cambio de residencia al nuevo Estado. Los artículos 65 y 66 recogían también la necesidad, por parte del interesado, de aportar certificaciones de la institución competente, por un lado, a efectos de cómputo de familiares para el cálculo de la prestación y, por otro, certificación de la prestación reconocida, cuantía y período reconocido a efectos de mantener el derecho reconocido tras un cambio de Estado de residencia, así como el mecanismo para la autorización por parte del Estado de recepción. Por tanto, el Reglamento $\mathrm{n}^{\mathbf{o}} 4$ no aportaba al derecho derivado contenido novedoso en cuanto a desempleo, al contener simplemente disposiciones de carácter técnico o administrativo con respecto al contenido del Reglamento $\mathrm{n}^{\mathrm{o}} 3$.

El 16 de agosto de 1961, se aprobó el Reglamento n ${ }^{\circ} 15$ del Consejo, relativo a las primeras medidas para lograr la libre circulación de los trabajadores dentro de la Comunidad $^{29}$. Este Reglamento representaba la primera etapa en la aplicación del derecho a la libre circulación de trabajadores ${ }^{30}$. En su artículo 6 se regulaba la posibilidad de renovación de la autorización de trabajo, y la ampliación de las posibles ocupaciones a desempeñar, para aquellos trabajadores que hubiesen cubierto y acreditado determinados períodos de ocupación regular previa en ese Estado. El apartado 1 del artículo 7 establecía la equiparación a períodos de ocupación regular las ausencias de trabajo que no superasen los cuarenta días, así como los días de vacaciones y las ausencias por enfermedad, maternidad, accidente de trabajo o enfermedad profesional.

El apartado 2 de este mismo artículo establecía que, entre otros, el período de desempleo involuntario, debidamente acreditado por el servicio público de empleo, si bien no era considerado como período de ocupación regular a efectos de acreditar los períodos a los que hacía referencia el artículo 6, no afectaba a los períodos ya reconocidos. No obstante, la persona desempleada debía aceptar un nuevo trabajo en cuanto le fuese ofrecido por el servicio público de empleo y acorde a la legislación de ese Estado. La importancia de esta mención al desempleo radicaba, por un lado, en su equiparación de no actividad como el servicio militar o situaciones de larga enfermedad

\footnotetext{
${ }^{29}$ DO n $^{\circ} 57$ del 26/08/1961, p. 1073/61.

30 RODRÍGUEZ-PIÑERO distingue tres etapas claras en la aplicación de la libre circulación de trabajadores. El Reglamento $\mathrm{n}^{\circ} 15$ del Consejo y su Directriz n 57 constituyen, en su opinión, la primera etapa en la aplicación de la libre circulación. Para mayor profundidad, véase RODRÍGUEZ-PIÑERO y BRAVO-FERRER, Miguel, "Libre circulación de los trabajadores y Seguridad Social de los trabajadores migrantes en Comunidades Europeas", Revista de Estudios Regionales, 1982, Vol. IV, pp. 147-149. Por su parte, GÓMEZ MUÑOZ considera al Reglamento $n^{\circ} 15$ como el origen del cuerpo jurídico sobre el que se asienta la libre circulación de trabajadores. GÓMEZ MUÑOZ, José Manuel, "La libre circulación de trabajadores: retrospectiva y evoluciones en el contexto europeo actual", Ponencia presentada en el marco del Proyecto Nacional I+D+i DER2015-67342-R, Redireccionando el empleo hacia el autoempleo: precariedad de condiciones de trabajo y emergencia de la figura del emprendedor. Financiado por el Ministerio de Economía y Competitividad y el FEDER, 2017.
} 
y, por otro, porque garantizaba la conservación de derechos ya generados. Además, si la situación de desempleo no superaba los cuarenta días, en virtud de lo establecido en el apartado 1 del artículo 7, ese período de tiempo era considerado como de ocupación regular a efectos de poder consolidar períodos de ocupación.

Por otro lado, el Reglamento contenía también una mención expresa al desempleo en su artículo 17. Así, dentro de las acciones a desenvolver entre los Estados miembros, la Comisión y los Comités (consultivo y técnico) en relación con la oferta y demanda de empleo, en su letra b) les encomendaba la elaboración de estudios en materia de empleo y desempleo para el desarrollo de políticas que garantizasen la libre circulación de trabajadores dentro de la Comunidad.

Por su parte, el Reglamento $\mathrm{n}^{\circ}$ 38/64/CEE del Consejo, de 25 de marzo de 1964, relativo a la libre circulación de trabajadores dentro de la Comunidad ${ }^{31}$, mantenía las menciones al desempleo ya contenidas en el Reglamento $n^{0} 15^{32}$, con la única variación de un nuevo apartado 3, dentro del artículo 7, de simple contenido aclaratorio sobre lo contenido en los apartados 1 y 2 . Este Reglamento, considerado como la segunda etapa en la implantación de la libre circulación de trabajadores ${ }^{33}$, no contenía novedad alguna en materia de desempleo con respecto a la ya contemplada en su antecesor.

El Reglamento (CEE) no 1612/68 del Consejo, de 15 de octubre de 1968, relativo a la libre circulación de trabajadores dentro de la Comunidad ${ }^{34}$, garantizaba en su artículo 5 la misma asistencia en las oficinas de empleo, en relación con los propios nacionales, a los nacionales de otros Estados miembros que buscasen un empleo en el Estado receptor. A su vez, el artículo 7 garantizaba la igualdad de trato en cuanto a las condiciones de empleo y trabajo y, en concreto en materia de despido y de reintegración profesional o de nuevo empleo, si hubiera quedado en situación de desempleo ${ }^{35}$.

Con la intención de materializar la idea de colaboración entre Estados miembros, manifestada en varios apartados del texto del Reglamento, el artículo 13.1 encomendaba a los Estados miembros o a la Comisión, de manera individual o en colaboración, la promoción de estudios en materia de empleo y desempleo para garantizar la libre

\footnotetext{
${ }^{31} \mathrm{DO} \mathrm{n}^{\mathrm{o}} 62$ del 17/04/1964, p. 965/64.

${ }^{32} \mathrm{Si}$ bien la tendencia era eliminar progresivamente las condiciones que limitaban la ocupación de determinadas ofertas de empleo a nacionales de un determinado país, éstas se mantenían en determinadas circunstancias, como señala al respecto, LÁZARO SÁNCHEZ, José Luis, "La libre circulación de trabajadores y las dificultades de desplazamiento de los desempleados", Revista Derecho y Conocimiento, Universidad de Huelva, 2001, Volumen I, p. 415.

${ }^{33}$ Junto con su Directriz no 240. RODRÍGUEZ-PIÑERO y BRAVO-FERRER, Miguel, Op. Cit., 1982, p. 148.

${ }^{34}$ DO n ${ }^{\circ}$ L257/2 del 19/10/1968, pp. 77 y ss.

35 Para mayor profundidad sobre el alcance y contenido de la igualdad de trato en cuanto a las condiciones de empleo y trabajo en el Reglamento (CEE) n n 1612/68, FERRÉ SALAS, Javier, "La libre circulación de trabajadores asalariados y la protección del emigrante", Revista de Gestión Pública y Privada, 1996, nº 1, pp. 61-65.
} 
circulación de trabajadores dentro de la Comunidad. Con este Reglamento y su Directriz $n^{\circ} 360$, se cerraba la tercera etapa en la aplicación de la libre circulación de trabajadores, al tiempo que se creaban los instrumentos administrativos imprescindibles para su efectiva puesta en práctica ${ }^{36}$.

En paralelo con el Reglamento, la Directiva 68/360/CEE, de 15 de octubre, sobre supresión de restricciones al desplazamiento y a la estancia de los trabajadores de los Estados miembros y de sus familias dentro de la Comunidad ${ }^{37}$, garantizaba en su artículo 7, apartado 1, la tarjeta de estancia en período de validez a un trabajador por el simple hecho de que éste hubiese dejado de ocupar un empleo [...] porque se halle en situación de paro involuntario debidamente comprobada por la oficina de empleo competente, dando así un plus de protección al trabajador desempleado y garantizando el libre desplazamiento y estancia en el territorio de otro Estado.

A su vez, el Reglamento (CEE) n 1251/70 de la Comisión, de 29 de Junio de 1970, relativo al derecho de los trabajadores a permanecer en el territorio de un Estado miembro después de haber ejercido en él un empleo ${ }^{38}$, enumeraba en su artículo 2.1 las situaciones que generaban este derecho ${ }^{39}$. Entre ellas, no se recogía la situación de desempleo, si bien, en su artículo 4.2 permitía computar los períodos de paro involuntario como períodos de empleo a efectos de acreditar las situaciones a las que se refería el artículo 2.1.

En el año 1971 se aprobó el Reglamento (CEE) n n 1408/71, de 14 de junio de 1971, relativo a la aplicación de los regímenes de seguridad social a los trabajadores por cuenta ajena y a sus familias que se desplazan dentro de la Comunidad ${ }^{40}$, que venía a actualizar y sustituir al Reglamento $\mathrm{n}^{\mathbf{0}} 3$. Así se recogía en su considerando primero, justificando su necesidad en la conveniencia de proceder a una revisión general del Reglamento $n^{o} 3$ del Consejo sobre la seguridad social de los trabajadores migrantes, como consecuencia, por un lado, de la experiencia práctica de su aplicación desde

\footnotetext{
${ }^{36}$ RODRÍGUEZ-PIÑERO y BRAVO-FERRER, Miguel, Op. Cit., 1982, p. 148.

${ }^{37} \mathrm{DO}^{\circ}{ }^{\mathrm{L}} \mathrm{L} 257 / 13$, de 19 de octubre de 1968, p. 88.

${ }^{38} \mathrm{DO}^{\circ}{ }^{\mathrm{L}} \mathrm{L} 142 / 24$, de 30 de junio de 1970, p. 93.

39 Artículo 2.1 del Reglamento (CEE) n 1251/70: “Tendrá derecho a residir permanentemente en el territorio de un Estado miembro: a) el trabajador que, al término de su actividad, haya alcanzado la edad prevista por la legislación de ese Estado para hacer valer sus derechos a una pensión por vejez y que haya ocupado un empleo en dicho Estado durante los últimos doce meses, como mínimo, y haya residido en él de manera continuada desde al menos tres años; b) el trabajador que, habiendo residido sin interrupción durante más de dos años en el territorio de ese Estado, dejase la ocupación de un empleo asalariado como consecuencia de una incapacidad laboral permanente. No se requerirá ninguna condición en cuanto a la duración de residencia, si dicha incapacidad resultase de accidente de trabajo o de enfermedad profesional que dé derecho a una pensión, de la que será responsable, total o parcialmente una institución de ese Estado ; c) el trabajador que, después de tres años continuados de empleo y residencia en el territorio de ese Estado, ocupase un empleo asalariado en el territorio de otro Estado miembro, manteniendo su residencia en el territorio del primer Estado, al que regresará, en principio, todos los días o, al menos, una vez por semana".

${ }^{40}$ DO n ${ }^{\circ}$ L149/2, de 5 de julio de 1971, p. 98.
} 
1959 y, por otro, de las modificaciones introducidas en las diversas legislaciones nacionales. A continuación, el considerando segundo recogía la idea de que las normas de coordinación vigentes pueden ser, en su conjunto, desarrolladas y mejoradas, a la vez que simplificadas en cierta medida, sin prescindir de las diferencias que subsisten entre las legislaciones nacionales de seguridad social. En definitiva, en los considerandos del propio Reglamento (CEE) nº 1408/71 se manifestaba la necesidad de mejorar o desarrollar aquellos aspectos en los que el Reglamento $\mathrm{n}^{\mathbf{0}} 3$ no se había mostrado ágil en su aplicación práctica, al tiempo que se le daba cabida en el nuevo Reglamento a las modificaciones que se habían producido en las legislaciones nacionales. Por otro lado, también en los propios considerandos, el legislador comunitario quería dejar patente, nuevamente, la independencia entre los Estados miembros en relación con sus respectivas legislaciones nacionales ${ }^{41}$.

La cuestión del desempleo se abordaba en el Capítulo 6, formado por cinco artículos a través de los cuales se regulaban las cuestiones en relación con la totalización de los períodos de seguro o de empleo (artículo 67), los criterios para el cálculo de las prestaciones por desempleo (artículo 68), las condiciones y límites para la conservación del derecho a las prestaciones (artículo 69), la dinámica de las prestaciones y su reembolso (artículo 70) y la dinámica de la prestación por desempleo para aquellos trabajadores en situación de desempleo que residieran, mientras ocupaban su último empleo, en un Estado miembro distinto del Estado competente (artículo 71). Esta última situación, contemplada en el artículo 71, representaba la novedad más salientable respecto a su predecesor, el Reglamento $\mathrm{n}^{\circ} 3$, al incorporar de manera efectiva a este particular colectivo de trabajadores. Dada la exclusión expresa que el propio Reglamento $\mathrm{n}^{\mathrm{o}} 3$ había contemplado en su artículo $4.3^{42}$, y la posterior inclusión de estos colectivos en virtud de los Reglamentos no 36/63/CEE del Consejo, de 2 de abril de

\footnotetext{
${ }^{41}$ MIRANDA BOTO, José María, "La Jurisprudencia reciente (2000-2005) del TJCE sobre desempleo y su influencia en la jurisprudencia española", en SÁNCHEZ-RODAS NAVARRO, Cristina (Dir.), Migrantes y Derecho, Murcia, Ediciones Laborum, 2006, p. 140: "El convencimiento de los Estados de que la protección del desempleo es una actividad muy íntimamente relacionada con el ejercicio de la soberanía estatal”, ARASTEY SAHÚN, Lourdes, “Aspectos polémicos en relación a la Seguridad Social en el Derecho Social Comunitario", en FALGUERA BARÓ, Miguel Ángel, MORALO GALLEGO, Sebastián. (Coord.), Derecho Social Europeo, Madrid, Centro de Documentación Judicial del Consejo General del Poder Judicial, 2006, p. 162: "Las dificultades para que se alcance una homogeneización en esta materia están muy lejos de poder ser superadas, pues, a las contundentes razones de tipo económico que separan los niveles de protección social en uno u otro Estado Miembro, se une, por un lado, el problema de los distintos conceptos de seguridad y protección social que se manejan en cada uno de ellos"; FERNÁNDEZ ORRICO, Francisco Javier, "La protección por desempleo en la Unión Europea a partir de la entrada en vigor de los Reglamentos (CE) 883/2004 y 987/2009”, Revista del Ministerio de Trabajo e Inmigración, 2010, $\mathrm{n}^{\circ}$ 89, pp. 99-100: "Cualquiera que lleve tiempo familiarizado con la normativa de la UE, sabe que tradicionalmente los Estados de la Unión han sido muy celosos dentro de su espacio territorial en la aplicación de sus normas internas, en particular en materia de Seguridad Social, existiendo gran reticencia en aceptar otras que provengan de terceros países. Esta fue, sin duda, la causa principal de que la Comunidad Europea partiera en sus orígenes desde el respeto a los sistemas nacionales".

42 Artículo 4.3 del Reglamento $\mathrm{n}^{\mathrm{o}}$ 3: "Las disposiciones de este Reglamento no se aplican a los trabajadores fronterizos ni a los trabajadores de temporada [...].
} 
$1963^{43}$ y n $^{\text {o } 73 / 63 / C E E ~ d e l ~ C o n s e j o, ~ d e ~} 11$ de julio de $1963^{44}$, el Reglamento (CEE) $\mathrm{n}^{\mathrm{o}}$ 1408/71 venía así a acoger en su artículo 71 las disposiciones particulares aplicables a estos trabajadores en materia de desempleo.

Con la misma función y en sustitución del antiguo Reglamento $\mathrm{n}^{\mathrm{o}}$ 4, nacía el Reglamento (CEE) no 574/72 del Consejo de 21 de marzo de 1972 por el que se establecían las modalidades de aplicación del Reglamento (CEE) n ${ }^{\circ}$ 1408/71 relativo a la aplicación de los regímenes de seguridad social a los trabajadores por cuenta ajena y a sus familiares que se desplacen dentro de la Comunidad ${ }^{45}$. En su Capítulo 6, establecía los criterios de aplicación de las cuestiones relativas a las prestaciones por desempleo contempladas en el mismo capítulo del Reglamento (CEE) no 1408/71. Al igual que su antecesor, el nuevo Reglamento recogía la necesidad de certificación de períodos de seguro o de empleo cubiertos bajo la legislación de otros Estados a efectos de totalización de períodos (artículo 80), igual certificación a efectos del cálculo de las prestaciones (artículo 81) y a efectos del cómputo de los miembros de la familia del interesado a tener en cuenta para el cálculo (artículo 82). Finalmente, el artículo 83 contenía las condiciones de aplicación del derecho a la conservación de las prestaciones cuando el desempleado se desplazaba a otro Estado miembro.

Consecuencia lógica de la novedad introducida por el Reglamento (CEE) nº 1408/71 en su artículo 71, el Reglamento (CEE) 574/72 incluía también un novedoso artículo 84 que contenía las disposiciones aplicables a los trabajadores desempleados que residieran, mientras ocupaban su último empleo, en un Estado miembro distinto del Estado competente. Su contenido se limitaba a disposiciones certificación y/o información de la situación del trabajador a efectos de la prestación por desempleo.

La presencia del desempleo en el derecho derivado de esta etapa, además de a través de Reglamentos y Directivas, también tuvo presencia por medio de otros instrumentos, las más de las veces Decisiones de la Comisión Administrativa ${ }^{46}$, generalmente aclaratorias o como ampliación de lo establecido en los anteriores ${ }^{47}$. También a través de Recomendaciones a los Estados, como la Recomendación de la Comisión 77/646/CEE, de 6 de julio, relativa a la preparación profesional de los jóvenes en paro o amenazados

\footnotetext{
${ }^{43}$ Reglamento $n^{\circ}$ 36/63/CEE del Consejo, de 2 de abril de 1963, relativo a la Seguridad Social de los trabajadores fronterizos. DO No 62, de 20 de abril de 1963.

${ }^{44}$ Reglamento $\mathrm{n}^{\mathrm{o}}$ 73/63/CEE del Consejo, de 11 de julio de 1963, que modifica e integra algunas disposiciones de los reglamentos $\mathrm{n}^{\mathbf{o}} 3 \mathrm{y}^{\mathbf{o}} 4$ relativo a los trabajadores de temporada y otros trabajadores que no residen en el país al que están sujetos. DO No 112, de 24 de julio de 1963.

${ }^{45}$ DO n ${ }^{\circ}$ L74/1, del 27 de marzo de 1972, p. 156.

${ }^{46}$ Por ejemplo, la Decisión $n^{\circ} 83$, de 22 de febrero de 1973, relativa a la interpretación del apartado 2 del artículo 68, del Reglamento (CEE) $n^{\circ}$ 1408/71 y del artículo 82 del Reglamento (CEE) $\mathrm{n}^{\circ}$ 574/72, referentes a los complementos de prestaciones de desempleo por cargas familiares. DO n ${ }^{\circ} \mathrm{C} 75 / 14$, de 19 de septiembre de 1973.

${ }^{47}$ Por ejemplo, la Decisión n ${ }^{\circ}$ 94, de 24 de enero de 1974, relativa al alcance del inciso ii) del punto b) del apartado 1, del artículo 71 del Reglamento (CEE) $\mathrm{n}^{\circ}$ 1408/71 del Consejo referente al derecho a las prestaciones de desempleo de los trabajadores distintos de los trabajadores fronterizos que, en el curso del último empleo, residieran en el territorio de un Estado miembro distinto del Estado competente.
} 
de perder su empleo que, si bien no abordaba la cuestión del desempleo, sí manifestaba en su exposición de motivos la preocupación de los Estados por esta cuestión y establecía recomendaciones de políticas activas de empleo (preparación profesional) dirigidas a los jóvenes ${ }^{48}$.

En el año 1978, vio la luz la Directiva 79/7/CEE del Consejo, de 19 de diciembre de 1978, relativa a la aplicación progresiva del principio de igualdad de trato entre hombres y mujeres en materia de Seguridad Social ${ }^{49}$, y que nacía como consecuencia de la incipiente preocupación por las discriminaciones por razón de sexo que existían en determinados aspectos de las legislaciones nacionales ${ }^{50}$. La creciente presencia de la mujer en los ámbitos profesionales hacía necesaria la adecuación de unos sistemas de protección social fundamentados en un mercado laboral de marcado carácter masculino $^{51}$. La aprobación de la Directiva 79/7/CEE perseguía la eliminación de cualquier tipo de discriminación por razón de sexo en el ámbito laboral con carácter general y, en particular, en cuestiones como cotizaciones, derecho y mantenimiento de prestaciones, etc. En relación con el desempleo, y tal y como recogía en su artículo 3, esta directiva resultaba aplicable a los regímenes legales que aseguren una protección contra los siguientes riesgos: [...] desempleo. Como ya se ha indicado, por medio de esta Directiva se garantizaba la igualdad de trato, entre otras, en las cuestiones relacionadas con el ámbito de aplicación de los regímenes, la obligación de contribuir y el cálculo de estas contribuciones, el cálculo de las prestaciones, etc.

La Resolución del Consejo de 27 de junio de 1980, sobre orientaciones para una política comunitaria del mercado de trabajo $^{52}$, aunque no abordaba cuestiones relativas al desempleo, sí contenía importantes resoluciones a los Estados en materia de readaptación profesional y la efectividad en la colocación de los servicios públicos de empleo.

Siguiendo la vía de las resoluciones, el Consejo adoptó una nueva el 12 de julio de 1982 sobre una acción comunitaria para combatir el desempleo ${ }^{53}$. Si bien no se establecían acciones concretas sobre la cuestión del desempleo, la Resolución comenzaba manifestando la preocupación del Consejo por el elevado nivel de paro, en particular entre los jóvenes, definiendo esta situación como intolerable. A continuación, disponía

\footnotetext{
${ }^{48} \mathrm{DO}^{\circ}{ }^{\mathrm{L}} \mathrm{L} 180 / 18$, de 20 de julio de 1977, p. 133.

${ }^{49} \mathrm{DO}^{\circ}{ }^{\mathrm{L}} \mathrm{L} 6 / 24$, de 10 de enero de 1979, p. 174.

50 Sobre esta creciente preocupación y la paulatina asunción de la nueva realidad laboral, ORTIZ LALLANA, Carmen, "Igualdad de derechos y oportunidades entre el hombre y la mujer en la Unión Europea", Revista del Ministerio de Trabajo y Asuntos Sociales, 2003, n 47, pp. 99-100; ALARCÓN CARACUEL, Manuel Ramón, "El principio de igualdad en el Derecho de la Unión Europea", en AAVV, La igualdad de trato en el Derecho Comunitario Laboral, Pamplona, Aranzadi, 1997, pp. 19 y ss.

${ }^{51}$ Para mayor profundidad sobre el origen, contenido y alcance de la Directiva 79/7/CEE, GUERRERO PADRÓN, Thais, "Directivas comunitarias sobre el principio de igualdad de trato entre hombres y mujeres y Seguridad Social", Revista Temas Laborales, 2003, nº 68, pp. 61-67.

${ }^{52} \mathrm{DO} \mathrm{n}^{\circ} \mathrm{C} 168 / 1$, de 8 de julio de 1980, p. 211.

${ }^{53} \mathrm{DO} \mathrm{n}^{\circ} \mathrm{C} 186 / 1$, de 21 de julio de 1982; p. 20.
} 
una serie de orientaciones dirigidas a los Estados miembros y en favor de una inversión pública y privada que tuviera en cuenta la repercusión de ésta en el empleo, la utilización de los instrumentos comunitarios en las regiones más afectadas por el paro, la reconversión de trabajadores y la adecuación de mano de obra cualificada, el desarrollo de la pequeña y mediana empresa, la formación profesional a los jóvenes, la movilidad geográfica y profesional, etc. Finalmente, la Resolución invitaba a la Comisión a la presentación de propuestas en el marco de una perspectiva comunitaria coordinada para combatir el desempleo. En el mismo sentido, la Resolución del Consejo de 11 de julio de 1983 sobre las políticas de formación profesional en la Comunidad Europea para la década de los 80 hacía hincapié en la formación profesional como vía para contrarrestar los elevados niveles de desempleo ${ }^{54}$.

También el Fondo Social Europeo, como instrumento de política de empleo, fue objeto de reformulación a través de la Decisión del Consejo de 17 de octubre de 1983 sobre las Funciones del Fondo Social Europeo ${ }^{55}$. Concebido como un instrumento financiero a través del cual la Comunidad desarrollaba sus acciones en materia social y de empleo, éste debía adaptarse a la cambiante realidad del mercado laboral europeo ${ }^{56}$. A través de esta Decisión del año 1983, el Consejo ponía más énfasis en las funciones del FSE encaminadas a la formación y orientación profesional, ayudas para el fomento del empleo, ayudas para la movilidad geográfica, asesoramiento técnico en materia de empleo, etc. En concreto, se ponía especial énfasis en acciones encaminadas a la formación de los jóvenes menores de veinticinco años o a aquellas otras personas que, aún superando esa edad, estaban incluidas en determinados colectivos como personas en paro o parados de larga duración, personas minusválidas, mujeres con interés en reanudar su vida profesional, trabajadores migrantes, etc ${ }^{57}$. Además, se incorporaba un criterio territorial en el destino de las acciones, reconociendo como zonas prioritarias, por ejemplo, países como Grecia o Irlanda.

En el año 1986 se publicó la Directiva del Consejo 86/378/CEE, de 24 de julio de 1986, relativa a la aplicación del principio de igualdad de trato entre hombres y mujeres en los regímenes profesionales de Seguridad Social ${ }^{58}$, secuela y complemento de la anterior Directiva 79/7/CEE ${ }^{59}$. En su artículo 4 asumía su aplicación a los regímenes

\footnotetext{
${ }^{54}$ DO n ${ }^{\circ}$ C193/2, de 20 de julio de 1983, p. 45.

${ }^{55}$ Decisión 83/156/CEE, de 17 de octubre de 1983 sobre las funciones del Fondo Social Europeo. DO n ${ }^{\circ}$ L289/38 de 22 de octubre de 1983, p. 26.

${ }^{56}$ MARTín VALVERDE, Antonio, El Fondo Social y la política de empleo en la Comunidad Europea, Bilbao, La Ley, 1986, pp. 29 - 46.

57 DOMÍNGUEZ GARRIDO, José Luis, La reforma de 1983 del Fondo Social Europeo, Madrid, Ministerio de Trabajo y Seguridad Social, Informes y Documentos, 1985, pp. 16-22; FRAILE, José Manuel, "El Fondo Social Europeo", Cuaderno de Relaciones Laborales, Madrid, Editorial Complutense, $1994, n^{\circ} 4$, p. 63.

${ }^{58} \mathrm{DO}^{\circ}{ }^{\mathrm{L}} \mathrm{L} 225 / 40$, de 12 de agosto de 1986.

${ }^{59}$ Sobre los matices entre regímenes legales y regímenes profesionales de Seguridad Social que daban lugar a estar amparados por una u otra directiva, GUERRERO PADRÓN, Thais, Op. Cit., 2003, pp. 67 68 .
} 
profesionales contra determinados riesgos, entre ellos, la protección por desempleo. En ese mismo año, se publicaba también la Resolución del Consejo 86/C 340/02, de 22 de diciembre de 1986 sobre el programa de acción para el incremento del empleo y que desarrollaba las líneas de acción de los Estados para el incremento del empleo con medidas dirigidas a la promoción de nuevas empresas y del crecimiento del empleo, la creación de mercados de trabajo más eficientes, la formación profesional o medidas para combatir el desempleo prolongado (apartado II, punto 4$)^{60}$.

Con fecha de 30 de diciembre de 1989 se publicó la Resolución del Consejo 89/C $328 / 01$, relativa a la creación de un observatorio europeo del empleo ${ }^{61}$. En ella se invitaba a la Comisión y a los Estados miembros a la creación de un observatorio en materia de empleo al que, entre otras, le asignaba la misión de analizar de forma permanente la evolución del empleo dentro de la Comunidad. Ese mismo año fueron publicados dos Reglamentos que modificaban determinados aspectos contenidos en los Reglamentos 1408/71 y 574/72, si bien dichos Reglamentos no contenían modificaciones que afectasen a la cuestión del desempleo ${ }^{62}$.

En mayo de 1990, el Consejo adoptó la Resolución 90/C 157/03, de 29 de mayo, relativa a una acción de asistencia a los parados de larga duración en la que, asumiendo la gravedad del desempleo de larga duración, proponía acciones conjuntas (a todos los niveles estatales y comunitarios, incluyendo a empresarios y agentes sociales) en materia de asesoramiento, formación profesional, medidas de estabilidad en el empleo, etc ${ }^{63}$.

A través de la modificación introducida por el Reglamento (CEE) n 2195/91, de 25 de junio de $1991^{64}$, se completaba el artículo 45 del Reglamento (CEE) n ${ }^{\circ}$ 1408/71 en lo concerniente a la consideración de los períodos de desempleo total a efectos del cómputo de los períodos de seguro o de residencia cubiertos bajo las legislaciones a que

\footnotetext{
${ }^{60} \mathrm{DO} \mathrm{n}^{\mathrm{o}} \mathrm{C} 340 / 2$ de 31 de diciembre de 1986.

${ }^{61} \mathrm{DO} \mathrm{n}^{\circ} \mathrm{C} 328 / 1$, de 30 de diciembre de 1989.

62 Reglamento (CEE) n $n^{\circ}$ 2332/89 del Consejo, de 18 de julio de 1989, por el que se modifican el Reglamento (CEE) $n^{\circ}$ 1408/71 relativo a la aplicación de los regímenes de seguridad social a los trabajadores por cuenta ajena, a los trabajadores por cuenta propia y a sus familias que se desplazan dentro de la Comunidad, y el Reglamento (CEE) $n^{\circ} 574 / 72$ por el que se establecen las modalidades de aplicación del Reglamento (CEE) $n^{\circ}$ 1408/71. DO n ${ }^{\circ}$ L224/1, de 2 de agosto de 1989; Reglamento (CEE) $\mathrm{n}^{\mathrm{o}} 3427 / 89$ del Consejo, de 30 de octubre de 1989, por el que se modifica el Reglamento (CEE) $\mathrm{n}^{\circ}$ 1408/71 relativo a la aplicación de los regímenes de seguridad social a los trabajadores por cuenta ajena, a los trabajadores por cuenta propia y a sus familias que se desplazan dentro de la Comunidad y el Reglamento (CEE) $n^{\circ} 574 / 72$ por el que se establecen las modalidades de aplicación del Reglamento (CEE) $n^{\circ} 1408 / 71$. DO n ${ }^{\circ}$ L331/1, de 16 de noviembre de 1989.

${ }^{63} \mathrm{DO}^{\circ}{ }^{\circ} \mathrm{C} 157 / 4$, de 27 de junio de 1990.

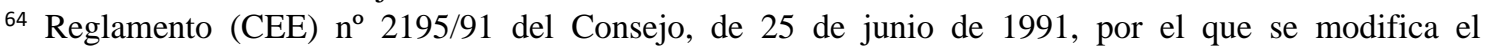
Reglamento (CEE) $n^{\circ}$ 1408/71 relativo a la aplicación de los regímenes de seguridad social a los trabajadores por cuenta ajena, a los trabajadores por cuenta propia y a sus familiares que se desplazan dentro de la Comunidad, y el Reglamento (CEE) $n^{\circ} 574 / 72$ por el que se establecen las modalidades de aplicación del Reglamento (CEE) nº 1408/71. DO nº L206/2, de 29 de julio de 1991.
} 
hubiera estado sujeto el trabajador para la adquisición, la conservación o la recuperación del derecho a prestaciones.

El Reglamento (CEE) no 1249/92 del Consejo ${ }^{65}$, del 30 de abril de ese mismo año, introducía una pequeña adaptación del apartado 9 del artículo 94 del Reglamento (CEE) n ${ }^{\circ}$ 1408/71 aplicable sólo a los trabajadores por cuenta ajena que en noviembre de 1989 percibían prestaciones por desempleo al amparo de la legislación francesa.

Por su parte, la Recomendación del Consejo 92/441/CEE ${ }^{66}$, de 24 de junio, sobre los criterios comunes relativos a recursos y prestaciones suficientes en los sistemas de protección social hacía extensibles sus recomendaciones, entre otras, a las cuestiones de empleo y desempleo.

También en el año 1992, el Reglamento (CEE) no 1248/92 del Consejo ${ }^{67}$, de 30 de abril, arrojaba un poco más de luz sobre las normas de cómputo de los períodos de seguro o residencia cumplidos en dos o varios Estados miembros a efectos de la prestación por esta contingencia.

En diciembre de 1992, el Consejo volvía a manifestar públicamente su preocupación por el desempleo a través de la Resolución del Consejo 93/C 49/02, de 21 de diciembre, relativa a la necesidad de abordar el grave problema del desempleo y el empeoramiento del mismo en la Comunidad ${ }^{68}$.

Un año después nacía la Red EURES de la mano de la Decisión de la Comisión 93/569/CEE ${ }^{69}$. La Red EURES se constituía como una red formada por los servicios de empleo de los distintos Estados miembros para el intercambio de información, cooperación y servicios en materia de búsqueda de empleo.

La continua preocupación del Consejo sobre los elevados niveles de desempleo en Europa propició en el año 1993 una nueva Resolución relativa al cometido de los sistemas de protección social en la lucha contra el desempleo en la que se instaba a los

65 Reglamento (CEE) $\mathrm{n}^{\mathrm{o}}$ 1249/92 del Consejo, de 30 de abril de 1992, por el que se modifica el Reglamento (CEE) $n^{\circ}$ 1408/71 relativo a la aplicación de los regímenes de seguridad social a los trabajadores por cuenta ajena, a los trabajadores por cuenta propia y a sus familias que se desplazan dentro de la Comunidad, y el Reglamento (CEE) n ${ }^{\circ} 574 / 72$ por el que se fijan las modalidades de aplicación del Reglamento (CEE) n 1408/71. DO nº L136/28, de 19 de mayo de 1992.

${ }^{66} \mathrm{DO} \mathrm{n}^{\circ} \mathrm{L} 245 / 46$, de 26 de agosto de 1992.

67 Reglamento (CEE) $n^{\circ} 1248 / 92$ del Consejo, de 30 de abril de 1992, por el que se modifica el Reglamento (CEE) $n^{\circ}$ 1408/71 relativo a la aplicación de los regímenes de seguridad social a los trabajadores por cuenta ajena, a los trabajadores por cuenta propia y a los miembros de sus familias que se desplacen dentro de la Comunidad, y el Reglamento (CEE) $\mathrm{n}^{\circ}$ 574/72 por el que se fijan las modalidades de aplicación del Reglamento (CEE) n 1408/71. DO nº L136/7, de 19 de mayo de 1992.

${ }^{68} \mathrm{DO} \mathrm{n}^{\circ} \mathrm{C} 49 / 3$ de 19 de febrero de 1992.

69 Decisión de la Comisión (93/569/CEE), de 22 de octubre de 1993, relativa a la aplicación del Reglamento (CEE) no 1612/68 del Consejo relativo a la libre circulación de los trabajadores dentro de la Comunidad, en lo que respecta, en particular, a una red creada bajo la denominación EURES (European Employment Services). DO n ${ }^{\circ}$ L274/32 de 6 de noviembre de 1993. 
Estados miembros a que incorporasen en sus políticas de protección social la lucha contra el desempleo con carácter general y, en concreto, que favorecieran la reinserción económica y social de los desempleados, que se abordase el problema del trabajo ilegal, etc ${ }^{70}$. Tres años más tarde se crearía el Comité de empleo y mercado de trabajo, por la vía de una Decisión del Consejo de 20 de diciembre de 1996, con la finalidad de analizar la evolución del empleo, las políticas de empleo y el mercado de trabajo en general y como vía de intercambio de información y experiencias de empleo entre los Estados miembro ${ }^{71}$.

Por la vía de dos Reglamentos, el Fondo Social Europeo sufriría en el año 1999 dos modificaciones en cuanto a sus objetivos y funciones en materia de empleo, ambas centradas en la mejora de la formación para el empleo y el acceso al mercado de $\operatorname{trabajo}^{72}$.

El 30 de abril del 2004 se publicaba en el Diario Oficial de las Comunidades Europeas el Reglamento (CE) nº 883/2004 del Parlamento Europeo y del Consejo de 29 de abril de 2004 sobre la coordinación de los sistemas de Seguridad Social, que venía a sustituir, completar y actualizar, en parte, al Reglamento 1408/71 ${ }^{73}$. La cuestión del desempleo se abordaba en el Capítulo 6. De manera casi simétrica a su antecesor, se regulaban las normas especiales sobre totalización de períodos de seguro, de empleo o de actividad (artículo 61), los criterios para el cálculo de las prestaciones (artículo 62), la operativa de la prestación en las situaciones de desplazamiento del desempleado a otros Estados (artículo 64) o los criterios para la determinación de la institución competente para el reconocimiento o pago de las prestaciones en supuestos concretos (artículo 65). Además de la eliminación del término fronterizo en la redacción del artículo, se introducían novedades en relación con las opciones de puesta a disposición frente a los servicios públicos de empleo por parte del desempleado, así como de los criterios de determinación de la institución competente.

Por medio de la Directiva 2006/54/CE del Parlamento Europeo y del Consejo ${ }^{74}$, de 5 de julio de 2006, relativa a la aplicación del principio de igualdad de oportunidades e igualdad de trato entre hombres y mujeres en asuntos de empleo y ocupación se producía la refundición de las Directivas de igualdad de trato entre hombres y mujeres publicadas con anterioridad. Al igual que sucedía con las anteriores, y tal y como se

\footnotetext{
${ }^{70}$ Resolución del Consejo (96/C 382/02) y de los Representantes de los gobiernos de los Estados miembros reunidos en el seno del Consejo de 2 de diciembre de 1996 relativa al cometido de los sistemas de protección social en la lucha contra el desempleo. $\mathrm{DO} \mathrm{n}^{\circ} \mathrm{C} 386 / 3$, de 20 de diciembre de 1996.

${ }^{71}$ Decisión del Consejo (97/16/CE), de 20 de diciembre de 1996, por la que se crea el Comité de empleo y del mercado de trabajo. DO L6/32 de 10 de enero de 1997.

${ }^{72}$ Reglamento (CE) no 1784/1999 del Parlamento Europeo y del Consejo, de 12 de julio de 1999, relativo al Fondo Social Europeo. DO L213/5 de 13 de agosto de 1999 y Reglamento (CE) $n^{\circ}$ 1262/1999 del Parlamento Europeo y del Consejo, de 21 de junio de 1999, relativo al Fondo Social Europeo. DO no L161/48 de 26 de junio de 1999.

${ }^{73}$ DO L166/1 de 30 de abril de 2004.

${ }^{74}$ DO L 204/28, de 26 de julio de 2006.
} 
recogía en su artículo 7.1.a), apartado $\mathrm{V}$, sería de aplicación a los regímenes profesionales de seguridad social que aseguren una protección contra los siguientes riesgos: [...]v) desempleo.

Cinco años después ${ }^{75}$ de la publicación del Reglamento (CE) no $883 / 2004$, se publicaba el Reglamento (CE) no 987/2009 del Parlamento Europeo y del Consejo, de 16 de septiembre de $2009^{76}$, por el que se adoptaban las normas de aplicación del Reglamento (CE) no 883/2004, sobre la coordinación de los sistemas de Seguridad Social. Este Reglamento de aplicación nacía con la intención de completar y sustituir al antiguo Reglamento (CEE) $n^{\circ} 574 / 72$. En su Capítulo 5 regulaba lo concerniente a las prestaciones por desempleo (artículos 54 a 57). Al igual que sucedía en la redacción de su predecesor, lo contenido en los artículos de este capítulo se centraba en aspectos formales de certificación de determinadas cuestiones a efectos del reconocimiento y pago de las prestaciones, la totalización de períodos, los desplazamientos de perceptores de prestaciones a otros Estados miembros, etc.

Nuevamente, en el año 2013, el Fondo Social Europeo volvía a ser objeto de análisis y reformulación a través del Reglamento (UE) no 1304/2013 del Parlamento Europeo y del Consejo, de 17 de diciembre de 2013, relativo al Fondo Social Europeo y por el que se derogaba el Reglamento (CE) $n^{0} 1081 / 2006$ del Consejo ${ }^{77}$. En esta revisión, se abogaba por la puesta en marcha de acciones que favorecieran el acceso al empleo de las personas desempleadas, la potenciación del empleo por cuenta propia, la modernización del mercado de trabajo, la igualdad en el acceso al empleo, etc.

La Directiva 2014/36/UE del Parlamento Europeo y del Consejo ${ }^{78}$, de 26 de febrero de 2014, sobre las condiciones de entrada y estancia de nacionales de terceros países para fines de empleo como trabajadores temporeros, garantizaba a estos trabajadores derechos en relación con el empleo y desempleo, tales como los beneficios de la protección social regulada en el artículo 3 de Reglamento (CE) n ${ }^{\circ}$ 883/2004, los servicios de asesoramiento sobre trabajos de temporada prestados por las oficinas de empleo, etc. Por su parte, la Directiva 2014/54/UE del Parlamento Europeo y del Consejo $^{79}$, de 16 de abril de 2014, sobre medidas para facilitar el ejercicio de los derechos conferidos a los trabajadores en el contexto de la libre circulación de los trabajadores, garantizaba en su artículo 2 el acceso al empleo y a la asistencia que

75 CARDENAL CARRO y HIERRO HIERRO ponen el foco en los órganos implicados y en la complejidad de determinadas materias para justificar su compleja tramitación y demora. En detalle, CARDENAL CARRO, Miguel, HIERRO HIERRO, Francisco Javier, "Movimientos migratorios y Seguridad Social: el esperado Reglamento de Aplicación", Revista Aranzadi Social, 2009, no 15, pp. 1126.

${ }^{76}$ DO L284/1, de 30 de octubre de 2009.

77 DO L347/470, de 20 de diciembre de 2013.

${ }^{78}$ DO L94/375, de 28 de marzo de 2014.

${ }^{79}$ DO L128/8, de 30 de abril de 2014. 
prestaban las oficinas de empleo, como medida indirecta para evitar el incremento del desempleo.

Ya en el año 2016, la Red EURES fue objeto de revisión y ampliación a través del Reglamento (UE) 2016/589 del Parlamento Europeo y del Consejo, de 13 de abril, relativo a una red europea de servicios de empleo, al acceso de los trabajadores a los servicios de movilidad y a la mayor integración de los mercados de trabajo ${ }^{80}$. A través de este Reglamento de restablecía y redefinía en funcionamiento de la Red EURES.

\section{Conclusiones}

A modo de conclusión, podría decirse que la principal vía de desarrollo en materia de desempleo en el ámbito del Derecho de la Unión Europea ha sido a través de instrumentos de Derecho derivado, mucho más adaptables a las distintas realidades de los Estados miembros. Los rígidos Tratados, de marcado carácter político y económico, y salvo alguna honrosa y limitada excepción, dejaron prácticamente al margen la cuestión social en general y la cuestión del desempleo en particular. En el mejor de los casos, la cuestión social se entendió como una consecuencia, a un segundo nivel, de la cuestión económica. La idea de que una mejora de la economía traería consigo una mejora social hizo que los esfuerzos normativos en el derecho originario se centrasen en la primera.

El uso de instrumentos de Derecho derivado ayudó, por un lado, a clarificar y garantizar determinados derechos en este ámbito y, por otro, a adaptarse a nuevas realidades del mercado laboral europeo. En relación con la cuestión del desempleo, mención aparte de los Reglamentos de coordinación, con disposiciones particulares aplicables al desempleo, las acciones en esta materia tomaron forma, mayormente, de políticas activas, centrando fundamentalmente su intervención en el fomento del empleo.

Dirigiendo la vista hacia el futuro, todo parece indicar que, a pesar de lo laborioso de un proceso que comenzó en diciembre de 2016, la esperada reforma del Reglamento (CE) $n^{\circ} 883 / 2004$ pronto incorporará nuevos elementos de interés en relación con la prestación por desempleo. El documento del Consejo de 25 de marzo de 2019 (7698/19 ADD 1 REV 1) avanza en este sentido aspectos del acuerdo provisional que, en materia de desempleo, se materializarán en la exigencia del cumplimiento en último lugar de un período mínimo de un mes de seguro, empleo o actividad por cuenta propia en el Estado miembro ante el que se solicita la prestación por desempleo para la totalización de períodos; el establecimiento de un período mínimo de exportación de la prestación de

\footnotetext{
${ }^{80}$ Reglamento (UE) 2016/589 del Parlamento Europeo y del Consejo de 13 de abril de 2016 relativo a una red europea de servicios de empleo (EURES), al acceso de los trabajadores a los servicios de movilidad y a la mayor integración de los mercados de trabajo y por el que se modifican los Reglamentos (UE) n 492/2011 y (UE) n 1296/2013. DO n ${ }^{\circ}$ L107/1, de 22 de abril de 2016.
} 
seis meses, con posibilidad de prórroga hasta el período máximo de prestación reconocida; o las novedades respecto a la determinación de la legislación aplicable a los trabajadores fronterizos, los criterios de puesta a disposición ante los servicios de empleo de los Estados de residencia o empleo, o las novedosas condiciones de exportación de la prestación aplicables a este colectivo de trabajadores.

Obviamente, España no será impermeable a estas modificaciones y tendrá que someterse a un proceso de reformulación interna que necesariamente deberá poner especial énfasis en las vías de colaboración administrativa entre Estados miembros; la revisión y actualización de los criterios de reconocimiento de exportación de la prestación por desempleo, así como de su hipotética prórroga; o los mecanismos de puesta a disposición y control de los trabajadores fronterizos ante los servicios de empleo españoles.

\section{Bibliografía}

ALARCÓN CARACUEL, Manuel Ramón, "El principio de igualdad en el Derecho de la Unión Europea", en AAVV, La igualdad de trato en el Derecho Comunitario Laboral, Pamplona, Aranzadi, 1997.

ARASTEY SAHÚN, Lourdes, "Aspectos polémicos en relación a la Seguridad Social en el Derecho Social Comunitario", en FALGUERA BARÓ, Miguel Ángel, MORALO GALLEGO, Sebastián. (Coord.), Derecho Social Europeo, Madrid, Centro de Documentación Judicial del Consejo General del Poder Judicial, 2006.

BLÁZQUEZ AGUDO, Eva María, "El futuro de la libre circulación de trabajadores. Repensando su contenido a partir de la Directiva Marco sobre mercado interior", Revista del Ministerio de Trabajo y Asuntos Sociales, 2006, nº 62.

CARDENAL CARRO, Miguel, HIERRO HIERRO, Francisco Javier, "Movimientos migratorios y Seguridad Social: el esperado Reglamento de Aplicación”, Revista Aranzadi Social, 2009, $\mathrm{n}^{\circ} 15$.

DOMÍNGUEZ GARRIDO, José Luis, La reforma de 1983 del Fondo Social Europeo, Madrid, Ministerio de Trabajo y Seguridad Social, Informes y Documentos, 1985.

FERNÁNDEZ ORRICO, Francisco Javier, "La protección por desempleo en la Unión Europea a partir de la entrada en vigor de los Reglamentos (CE) 883/2004 y 987/2009", Revista del Ministerio de Trabajo e Inmigración, 2010, $\mathrm{n}^{\circ} 89$. 
FERRÉ SALAS, Javier, "La libre circulación de trabajadores asalariados y la protección del emigrante", Revista de Gestión Pública y Privada, 1996, nº 1.

FRAILE, José Manuel, “El Fondo Social Europeo”, Cuaderno de Relaciones Laborales, Madrid, Editorial Complutense, 1994.

GÁRATE CASTRO, Javier, “Apuntes sobre la regulación y funcionamiento del Fondo Social Europeo", Revista Española de Derecho del Trabajo No 30, 1987, pp. 243-267.

GARCÍA MURCIA, Joaquin, "Desempleo", Ponencia General del XIV Congreso Nacional de Derecho del Trabajo y de la Seguridad Social, Madrid, Ministerio de Trabajo y Asuntos Sociales, Colección Informes y Estudios, Serie Relaciones Laborales, 2004, nº 61.

GÓMEZ MUÑOZ, José Manuel, "La libre circulación de trabajadores: retrospectiva y evoluciones en el contexto europeo actual", Ponencia presentada en el marco del Proyecto Nacional I+D+i DER2015-67342-R, Redireccionando el empleo hacia el autoempleo: precariedad de condiciones de trabajo y emergencia de la figura del emprendedor. Financiado por el Ministerio de Economía y Competitividad y el FEDER, 2017.

GUERRERO PADRÓN, Thais, "Directivas comunitarias sobre el principio de igualdad de trato entre hombres y mujeres y Seguridad Social", Revista Temas Laborales, 2003, $n^{\circ} 68$.

LAMBEAUX, O, «L'accord international de sécurité sociale applicable aux bateliers du Rhin », Revista Droit Social, 1957, n 9-10.

LÁZARO SÁNCHEZ, José Luis, "La libre circulación de trabajadores y las dificultades de desplazamiento de los desempleados”, Revista Derecho y Conocimiento, Universidad de Huelva, 2001, Volumen I.

MANGAS MARTÍN, Araceli, LIÑÁN NOGUERAS, Diego Javier, Instituciones y Derecho de la Unión Europea, Madrid, Editorial Mc Graw Hill, 1996.

MARTÍN VALVERDE, Antonio, El Fondo Social y la política de empleo en la Comunidad Europea, Bilbao, La Ley, 1986.

MIRANDA BOTO, José María, "La Jurisprudencia reciente (2000-2005) del TJCE sobre desempleo y su influencia en la jurisprudencia española”, en SÁNCHEZ-RODAS NAVARRO, Cristina (Dir.), Migrantes y Derecho, Murcia, Ediciones Laborum, 2006. 
NIELSEN, R, EU Labour Law, Copenhagen, DJOF Publishing, 2013.

ORTIZ LALLANA, Carmen, "Igualdad de derechos y oportunidades entre el hombre y la mujer en la Unión Europea”, Revista del Ministerio de Trabajo y Asuntos Sociales, $2003, n^{\circ} 47$.

RIBAS, Jacques Jean, JONCZY, Marie Jose, SÉCHÉ, Jean Claude, Derecho Social Europeo, Madrid, Instituto de Estudios Sociales, Ministerio de Trabajo, 1980.

RODRÍGUEZ-PIÑERO y BRAVO-FERRER, Miguel, "Libre circulación de los trabajadores y Seguridad Social de los trabajadores migrantes en Comunidades Europeas", Revista de Estudios Regionales, 1982, Vol. IV.

RODRÍGUEZ-PIÑERO y BRAVO-FERRER, Miguel, "La Carta Social Europea y su puesta en práctica”, Revista de Instituciones Europeas N No 5, 1978.

RODRÍGUEZ-PIÑERO ROYO, Miguel, CASTELLANO BURGUILLO, Emilia, "La política de empleo de la Unión Europea", Trabajo: Revista Andaluza de Relaciones Laborales $\mathrm{N}^{\mathrm{o}}$ 10, 2001.

SCIARRA, Silvana, "Social Values and the Multiple Sources of European Social Law", European Law Journal, 1995, Vol. 1, nº1. 